\title{
GESTÃO NAS ESCOLAS MÉDICAS E SUSTENTABILIDADE DOS PROGRAMAS DE DESENVOLVIMENTO DOCENTE
}

\author{
Maria Tereza Carvalho Almeida* \\ Fernanda Alves Maia** \\ NiLdo Alves Batista***
}

Recebido: 26 set. 2011

Aprovado: 8 fev. 2012

*Farmacêutica, Mestre em Ensino em Ciências da Saúde. Professora da Universidade Estadual de Montes Claros. Montes Claros, MG, Brasil. E-mail: tereza.farmaco@hotmail.com

** Bióloga. Mestre em Ginecologia, Obstetrícia e Mastologia com ênfase em Ciências da Saúde. Professora da Universidade Estadual de Montes Claros. Montes Claros, MG, Brasil. E-mail: falvesmaia@gmail.com

***Doutor em Medicina (Pediatria) pela Universidade Federal de São Paulo. Atualmente é Pró-Reitor Adjunto da Pró-Reitoria de Planejamento da Universidade Federal de São Paulo. São Paulo, SP, Brasil. E-mail:nbatista@unifesp.br

Resumo: O objetivo deste trabalho consistiu em conhecer a percepção dos docentes, que trabalham em escolas médicas que utilizam métodos ativos de ensino/aprendizagem sobre o desempenho e a importância da gestão na sustentabilidade dos Programas de Desenvolvimento Docente (PDD). Os sujeitos desta pesquisa foram 115 professores do curso de medicina de uma Universidade Estadual que trabalham com a Aprendizagem Baseada em Problemas (ABP) e Problematização e já desempenharam as diversas funções docentes propostas. Na coleta de dados utilizamos um questionário com assertivas relacionadas à temática pesquisada e análise do grau de concordância e/ou discordância que foram sistematizadas, classificadas, tabuladas e dispostas em gráficos. Para o aprofundamento da pesquisa, utilizamos a entrevista semi-estruturada, cujos dados foram submetidos à análise temática. Os resultados indicam fragilidades da gestão em relação à sustentabilidade do PDD. Os docentes ressaltam que este programa somente será efetivo se a gestão do curso garantir a sua sustentabilidade, o que implica assumir a sua importância, institucionalização, planejamento e apoio na implantação e execução. Eles não falam sobre a ação dos gestores dessa instituição no PDD, mas enfatizam sim o despreparo dos mesmos, as consequências dessa falta de preparo e assumem a necessidade do próprio preparo para assumir as funções de gestores propostas nos métodos ativos. Eles reconhecem a avaliação formativa como um importante instrumento de gestão, porém destacam que ela não tem sido utilizada de forma efetiva. No exercício de qualquer uma das suas atividades o gestor é constantemente avaliador, portanto, acredita-se que se a avaliação estivesse sendo utilizada pelos gestores como um instrumento formativo dos atores envolvidos e do processo de ensino/aprendizagem, nesta pesquisa, não teriam aparecido tantas fragilidades relativas à gestão, o que possivelmente permitiria maior sustentabilidade ao PDD. São necessários outros estudos que aprofundem esta discussão, uma vez que se trata de um tema de grande relevância e importância.

Palavras-chave: Gestão. Desenvolvimento docente. Educação médica. Aprendizagem baseada em problemas.

\section{MANAGEMENT IN MEDICAL SCHOOLS AND SUSTAINABILITY OF FACULTY DEVELOPMENT PROGRAMS}

Abstract: The aim of this study was to understand the perception of teachers who work in medical schools that use active methods of teaching / learning about the importance of management performance and sustainability of a Faculty Development Program (PDD). The subjects were 115 medical school teachers in a State University that work with Problem Based Learning (PBL) and Problematization and they have performed many of the roles proposed. The data collected used a questionnaire with 


\begin{abstract}
statements related to the topic researched and assess the degree of agreement and / or disagreement systematized, classified, tabulated and displayed on graphs. For further research, we used semi-structured interviews, and the data was submitted to thematic analysis. The results indicate weaknesses of management regarding the sustainability of the PDD. Teachers point out that this program will only be effective if the management of the course ensure its sustainability, thereby assuming its importance, institutionalization, planning and support in the implementation and execution. They do not talk about the actions of the managers of this institution in PDD but they do emphasize the unpreparedness of the team, the consequences of this lack of preparation and assumes the need to prepare itself to assume the functions proposed by the active methods. They recognize the formative evaluation as an important management tool and also emphasize that it has not been used effectively. In exercising any of its activities, the manager is constantly an evaluator, therefore, it is believed that if the assessment was being used by managers as an formative instrument of the actors involved and the process of teaching / learning, this research would not have appeared so many weakness in the management, which might allow greater sustainability to the PDD. It is necessary, other studies to broaden this discussion, since it is a topic of great relevance and importance.
\end{abstract}

Key words: Management. Development faculty. Medical education. Problem based learning.

\title{
INTRODUÇÃO
}

Nos últimos anos, importantes ações governamentais foram realizadas no sentido de revitalizar os valores humanísticos na saúde (ALMEIDA; BATISTA, 2011). Em 2001 foram promulgadas as Diretrizes Curriculares Nacionais (DCN's) para os cursos da área da saúde que apontaram para uma concepção mais ampla de saúde e estabeleceram como horizonte desejável para a organização dos cursos os currículos integrados, que possibilitassem a superação da organização disciplinar e a articulação de várias disciplinas em torno de temáticas relevantes e estimulantes (TSUJI; AGUILAR-DA-SILVA, 2010). Na tentativa de atender estas exigências, algumas escolas têm optado pela utilização de métodos ativos de ensino/aprendizagem priorizando a Aprendizagem Baseada em Problemas (ABP) e a Problematização, o que tem implicado em mudanças importantes na maneira de pensar, organizar e desenvolver seus cursos.

Apesar dessas estratégias de ensino/aprendizagem corroborarem com as DCN's, a simples mudança do currículo e das estratégias não garante a mudança de concepções e práticas dos professores (PERIM et al, 2009). Está nos docentes a força motriz para as mudanças desejadas, porém, ainda há um investimento insuficiente na formação destes e, consequentemente, um menor avanço para as transformações (IOCHIDA, 2004).

Dentre as 28 escolas participantes no projeto da Comissão de Avaliação das Escolas Médicas (CAEM), a maioria entende que as escolas não oferecem nem promovem a formação pedagógica do corpo docente; apóiam e/ou oferecem periodicamente a atualização técnico-científica dos docentes; procuram estimular os docentes a participar dos serviços, embora ainda de forma esporádica; 
e não promovem, mas reconhecem a importância da capacitação gerencial do corpo docente. Estes fatores tornam o eixo desenvolvimento docente, o mais distante do preconizado pelas DCN's (PERIM et al, 2009).

Faz-se necessário a institucionalização dos Programas de Desenvolvimento Docente (PDD), o que requer uma ação efetiva da gestão da escola. Venturelli e Fiorini (2001) destacam como atribuições do grupo gestor a organização de sistemas de apoio aos docentes, para sua avaliação acadêmica e planejamento de contratações; a proteção do "acervo profissional docente", o que implica estabelecer um programa de desenvolvimento acadêmico progressivo para melhorar o desempenho do docente na universidade. Almeida e Batista (2011) ressaltam a importância dos gestores oferecerem o apoio necessário para que os PDD aconteçam de forma sistemática e que sejam considerados nos critérios de promoção acadêmica.

Assim, torna-se pertinente conhecer a percepção dos docentes, que trabalham numa escola médica que utiliza métodos ativos de ensino/aprendizagem há 10 anos, sobre o desempenho e a importância da gestão na sustentabilidade do PDD.

\section{METODOLOGIA}

Trata-se de uma pesquisa exploratória, descritiva e analítica com abordagens quantitativa e qualitativa. A população de estudo consistiu de 115 docentes (63,2\% do total de professores) atuantes no curso de medicina da Universidade Estadual de Montes Claros/Unimontes, sendo nove (7,8\%) doutores, 36 (31,3\%) mestres e $70(60,9 \%)$ especialistas. Todos os docentes exerciam alguma atividade em um currículo com métodos ativos de ensino-aprendizagem e tinham vivenciado os impactos da mudança curricular (ocorrida em 2001).

Para a coleta de dados utilizamos um questionário fechado e uma entrevista semi-estruturada de aprofundamento. $\mathrm{O}$ uso de mais de uma técnica de coleta de dados permitiu aos pesquisadores combinar vantagens e corrigir algumas deficiências, ou seja, validar e aprofundar a representação das informações obtidas (PASQUALI, 1996).

Para a elaboração do questionário foram utilizadas quatro questões fechadas para caracterização da amostra (idade, tempo de docência, profissão e titulação acadêmica) e dez assertivas sobre o objeto de estudo as quais, numa escala de Likert, os sujeitos da pesquisa apontaram seus níveis de concordância/ discordância (BOGDAN; BIKLEN, 1994). As assertivas foram elaboradas explorando a percepção dos docentes sobre o papel e a importância da gestão na sustentabilidade dos PDD. 
O questionário foi aplicado durante as reuniões periódicas mensais de cada departamento e os dados obtidos foram sistematizados, classificados, tabulados e dispostos em gráficos.

Na segunda fase (entrevistas de aprofundamento), trabalhamos com 15 docentes selecionados por sorteio aleatório conforme funções desempenhadas no currículo: 3 tutores, 2 construtores, 4 preceptores, 3 instrutores, 1 professor da comissão de avaliação, 1 coordenador de período e 1 gestor membro da comissão de educação médica. Importante ressaltar que a maioria dos participantes nesta fase já tinha exercido pelo menos duas dessas funções.

O número de sujeitos desta etapa da pesquisa foi determinado em função dos dados qualitativos terem se mostrado "suficientes para realizar aquilo a que nos propusemos” (BOGDAN; BIKLEN, 1994, p. 200). Para Minayo (2004), nos pressupostos qualitativos o número de entrevistas torna-se suficiente à medida que se observa uma reincidência das informações.

As entrevistas foram realizadas seguindo um roteiro prévio determinado a partir das análises dos dados obtidos com o questionário sem limitar ou restringir a espontaneidade das respostas dadas pelos participantes no estudo, constando da seguinte pergunta norteadora: "Qual a sua percepção sobre o desempenho e a importância da gestão para o PDD?”. Foram gravadas e transcritas pelos pesquisadores respeitando-se as pausas e outras expressões que pudessem auxiliar a tradução da realidade. Após sua transcrição, os dados foram sistematizados e submetidos à análise temática, uma das técnicas de Análise de Conteúdo, valorizando os significados presentes nas falas, a sua correlação com as questões formuladas e a articulação com o marco teórico adotado na pesquisa.

Este estudo é parte de uma dissertação de mestrado desenvolvida junto à Universidade Federal de São Paulo/Unifesp (2009-2010), aprovado pelo Comitê de Ética da Universidade, em cumprimento da resolução n ${ }^{\circ}$ 196/96. Protocolo $n^{\circ}$ 0445/09. Os sujeitos assinaram o Termo de Consentimento Livre e Esclarecido, garantindo-se o sigilo dos dados coletados, bem como o direito às informações necessárias.

\section{RESULTADOS E DISCUSSÃO}

Os cursos de medicina têm optado pela utilização de métodos ativos de ensino/aprendizagem com o objetivo de atender às demandas das DCNs, bem como as demandas do Sistema Único de Saúde (SUS) vigente no país. Assim, os professores passam a assumir diversificadas atividades docentes. 
Quando questionados sobre a assertiva: “Os programas de desenvolvimento docente devem proporcionar aos docentes a formação para atuarem em todas as atividades propostas na $\mathrm{ABP}$, pois são eles que darão suporte ao desenvolvimento do projeto educacional", há concordância de $93,1 \%$ dos professores pesquisados. E, 91,2\% acreditam ser imprescindível estarem inseridos num programa de desenvolvimento docente, conforme pode ser verificado no gráfico abaixo:

\section{Gráfico 01 - Assertiva: Os métodos ativos de ensino/aprendizagem exigem um maior envolvimento/conhecimento do processo de ensinar e aprender, por isso é imprescindível ao docente estar inserido num programa de desenvolvimento docente.}

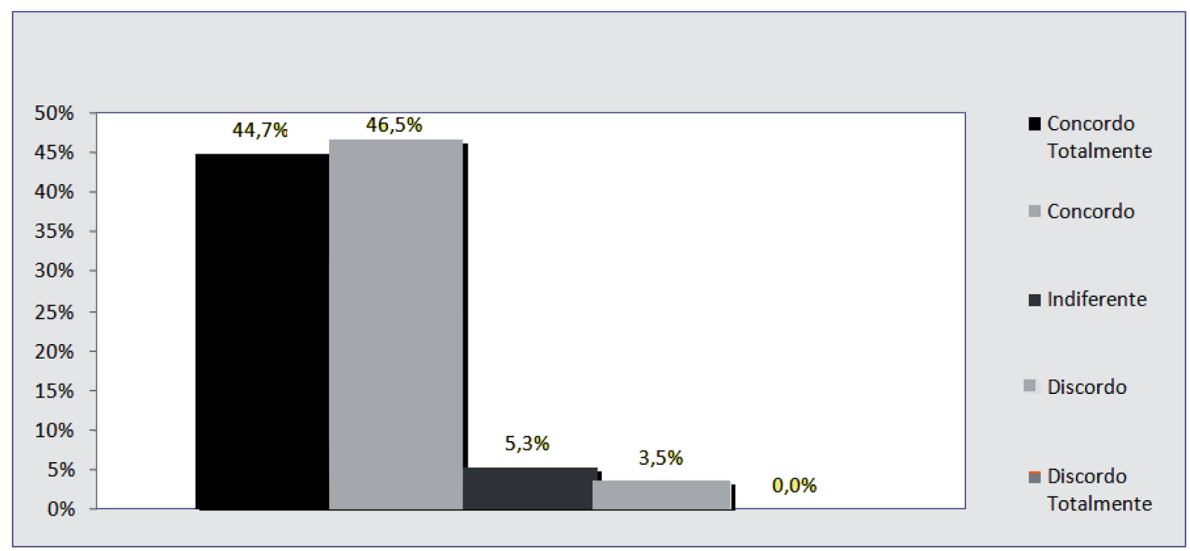

Wilkerson e Irby (1998), Venturelli e Fiorini (2001) confirmam a necessidade apontada pelos professores de ser oferecido aos docentes a oportunidade de se prepararem para atuar em todas as atividades propostas nos currículos que utilizam métodos ativos. Perim et al (2009) privilegiam o papel do docente não mais na função de ensinante, mas como protagonista das mudanças que se fazem urgentes e afirma que não há como pensar nas transformações nos cursos de medicina dissociadas do docente. Um professor assim se posiciona: [...] educação permanente, com aspectos diferenciados para tutor, instrutor de habilidades, instrutor de ambulatório, instrutor de internato (P3).

Outro aspecto relevante e que aparece nesta pesquisa se refere à necessidade de integração dos professores para se alcançar a integração dos conteúdos e compreensão das necessidades de saúde, conforme proposto pelo modelo da integralidade: [...] não adianta você querer integrar conteúdos se você não integra pessoas (P3). Este dado é confirmado por Silva (2006). 
Dentre as necessidades no processo de formação/desenvolvimento docente um grande destaque é dado à necessidade de investimento institucional como condição para que haja a indução e a sustentabilidade do PDD: [...] a universidade poderia nos ajudar nesse sentido porque [...] essa busca individual de desenvolvimento [...] é infrutifera (P6).

Cunha (1998), Venturelli e Fiorini (2001), Almeida et al (2003), Batista e Batista (2004), Campos et al (2007), Costa (2010) confirmam essa demanda e acrescentam que o desenvolvimento de práticas de formação/desenvolvimento docente em saúde deve estar inserido em um projeto institucional mais amplo, o que implica assumir a formação do professor como um processo permanente, em que seus saberes docentes vão se transformando à medida que empreende seu trabalho pedagógico, problematiza e investiga sua própria prática.

Perim et al (2009) reforçam a necessidade de apoio institucional para se desenvolver tanto no que se refere ao conhecimento técnico-científico e ao processo de ensino/aprendizagem, como também na gestão do curso médico. Nesse sentido, o professor universitário precisa de instrumentos teórico-metodológicos que estejam continuamente em debate, para que consiga redimensionar suas concepções e fazeres. As falas a seguir confirmam essa demanda e apontam as necessidades dos professores de estarem inseridos em PDD significativos, sistematizados, permanentes e institucionalizados: [...] a gente teria que ter instrumentos adequados, recursos que realmente fizessem a diferença, que fosse significativo para o docente sair da sua casa, sair do seu consultório, sair do seu trabalho, ir para lá, participar (P4); Que haja oficinas permanentes, trabalhando a área de atuação de cada um (P11).

Venturelli e Fiorini (2001) ressaltam a importância da especificidade dos PDD, uma vez que diferentes escolas têm diferentes problemas e diferentes necessidades. É pertinente respeitar essas diferenças e não buscar soluções padrão, o que requer um importante trabalho de análise, uma avaliação permanente e consequentemente uma gestão efetiva. As falas desses professores confirmam essas necessidades: Dentro do PBL (ABP) não dá para ser um programa único, porque as perspectivas docentes [...] são diferentes de acordo com as atividades que o docente está desenvolvendo (P3); Talvez dentro da coordenação, gestão [...] capacidade de trabalhar em equipe, aperfeiçoar mais [...] espírito de liderança, de saber como liderar o grupo (P9).

A gestão das escolas médicas implica no desenvolvimento de lideranças capazes de planejar e gerenciar o currículo, bem como garantir que as atividades docentes e discentes sejam cumpridas com motivação e os resultados esperados sejam alcançados (ALMEIDA; BATISTA, 2011). 
Almeida (2008) enfatiza que as perspectivas de gestão da escola dependem do sucesso obtido na formação de novas lideranças, preparadas para a gestão de mudanças. Para ele, a formação médica depende de boas práticas de gestão para o bom desenvolvimento das atividades acadêmico-administrativas e, especialmente, para alcançar sucesso nas mudanças almejadas. No entanto, a gestão na escola médica não é profissionalizada e não é reconhecida e valorizada pela comunidade acadêmica. A fala desse professor vai ao encontro desta afirmativa: O gestor [...] tem uma capacitação do tipo ser médico de tal especialidade, mas ele não tem capacitação para ser, para gerenciar e administrar grupos, pessoas, recursos humanos $(\mathrm{P} 4)$.

Ao realizar algumas críticas/autocríticas do processo de gestão na escola médica, Almeida (2008) enfatiza que, dentre outras questões, a auto suficiência dos médicos que mesmo após tomar consciência de suas deficiências, procuram resolvê-las por meio de apropriação personalizada dos conhecimentos de outras esferas de atuação; a dicotomia acadêmico/administrativa, mais presente nas instituições públicas e favorecidas pela força do pensamento biomédico e do paradígma flexneriano que ainda prevalece na sociedade; a ilusão de que a gestão da escola se resuma a ter planos anuais e de ação.

Venturelli e Fiorini (2001) afirmam ser essencial desenvolver cursos que subsidiem a formação dos futuros líderes das diferentes áreas. Os problemas de gerenciamento e cooperação, de integração e pesquisa precisam ser baseados num conhecimento real e prático do programa.

Os professores pesquisados reconhecem a importância do preparo dos gestores para que as mudanças propostas se efetivem e o curso de medicina alcance os resultados almejados. Entretanto, eles destacam a falta de preparo para o exercício da gestão: A gestão, os gestores, eles têm as suas limitações, que estão no plano institucional, têm suas limitações pessoais [...] essas limitações somadas [...] geram uma desmotivação, uma descrença [...] tem muito movimento, mas pouca mudança (P4). Também reconhecem as consequências dessa falta de preparo: O coordenador [...] acaba ficando muito numa função de resolver questões que não seriam ele e com isso ele envolve pouco com o curso, com a metodologia [...] a questão do currículo em si, do processo em si (P13). A falta de preparo dos gestores, bem como a falta de destaque dos professores pesquisados em relação à ação dos gestores no PDD indica uma fragilidade da gestão na sustentabilidade destes programas.

Quando questionados se sentem motivados pela equipe de coordenação do curso para melhorar sua competência para desempenhar as atividades docentes propostas, $27 \%$ afirmam que não e 25,3\% mostram-se indiferentes. O que nos 
faz pensar que os gestores não têm desempenhado de forma satisfatória uma das suas funções mais importantes: motivar sua equipe de docentes. As falas dos professores refletem este achado: A motivação da gestão, da coordenação [...] é quase insignificante (P6); Uns grupos ficam mais animados do que outros e depende muito do coordenador (P9).

Almeida (2008) reforça que, quando os gestores não dão a devida atenção às preocupações, dificuldades e demandas dos docentes, esses podem atuar de forma não cooperativa e comprometer os resultados esperados.

Ao se trabalhar com métodos ativos, os gestores contam com um importante instrumento de gestão: a avaliação formativa. Tsuji e Aguilar-da-Silva (2010) acrescentam que o processo de avaliação nos indica as fortalezas e fragilidades, não somente do estudante, mas também do processo de ensino/aprendizagem (tutoria, ciclo pedagógico, desempenho do tutor, problemas utilizados para discussão, articulação biopsicossocial, etc.). Os professores pesquisados assim a reconhecem: Eu sempre vou atrás da avaliação [...] como coordenadora de periodo. Isso tem uma influência [...] direta (P2). Uma vez que permeia todo o processo de ensino/aprendizagem, a avaliação pode ser utilizada para fins de diagnóstico, para orientar as tomadas de decisão que se fizerem necessárias, bem como orientar o PDD.

Professores e estudantes são convidados a realizá-la ao final de todas as atividades e registrá-las na intranet. Eles destacam a necessidade de se realizar os consolidados das avaliações da intranet, para que possam ser utilizados como instrumentos de gestão. Esses consolidados podem ser utilizados pelo PDD como estratégia de desenvolvimento docente: Tanto individualmente, para que ele tenha acesso aos seus dados individuais e pessoais e de forma coletiva, levantando resumos sobre avaliação dos docentes, mostrando para eles como está a sua evolução (P11). Assim, destacam a importância do feedback, o que de acordo com Rushton (2005) para que seja efetivo, faz-se necessário a motivação, a capacitação e a utilização de estratégias adequadas. Hoffmann (2008) acrescenta que ofeedback vai contribuir para o crescimento e para o desenvolvimento das potencialidades se a avaliação for significativa, e para isso os dados têm que ser verdadeiros e os instrumentos avaliativos coerentes e adequados. Entretanto, os professores pesquisados destacam que ainda persiste uma cultura avaliativa arraigada no medo e na imaturidade: [...] as avaliações não são verídicas, são [...] superficiais [...] tem muito medo de estar avaliando criticamente $(\mathrm{P} 1) . \mathrm{O}$ que nos leva a refletir que não houve investimento suficiente na capacitação das pessoas envolvidas. 
Dessa forma, o curso deve contar com um núcleo de avaliação que acompanhe o processo de ensino/aprendizagem e consequentemente os docentes, garantindo o diagnóstico e as orientações ao PDD. Tsuji e Aguilar-da-Silva (2010) acrescentam que ao constituir o seu núcleo, deve-se levar em consideração as particularidades individuais, o modelo curricular e o método pedagógico. Os professores pesquisados reconhecem a necessidade e a importância do núcleo de avaliação, porém demonstram que, em suas práticas ainda não tem funcionado efetivamente: [...] a avaliação do processo tutorial também é falha em função [...] da falta de uma comissão de avaliação (P13).

Os docentes reconhecem a importância da instituição oferecer o PDD adequado às demandas da própria instituição, mas também às suas demandas. Eles ressaltam que este programa somente será efetivo se a gestão der a sustentabilidade, o que implica assumir a sua importância, garantir sua institucionalização, planejamento, e apoio na implantação e execução. Entretanto, eles enfatizam o despreparo dos gestores e identificam algumas conseqüências. A falta de destaque dos professores pesquisados em relação à ação dos gestores no PDD indica uma fragilidade da gestão na sustentabilidade destes programas. Nesta perspectiva, eles destacam a necessidade dos gestores se prepararem e assumem a necessidade do próprio preparo para assumir as funções de gestores no curso.

Dessa forma, investigar a percepção dos docentes sobre o desempenho e a importância da gestão na sustentabilidade do PDD em métodos ativos de ensino/ aprendizagem nos leva a refletir sobre as diversificadas funções e os desafios enfrentados pelos gestores. O cata-vento a seguir demonstra a complexidade deste processo.

Esta figura nos remete à idéia de movimento e desenvolvimento que reforça o modelo que sustenta a utilização dos métodos ativos pelas escolas que buscam principalmente a permanente mudança de atitudes dos diversos atores envolvidos (gestores, docentes, estudantes, equipe técnica, etc.), as mudanças nos processos de ensino/aprendizagem e, consequentemente, a mudança na formação do médico (ALMEIDA; BATISTA, 2011).

$O$ gestor ganha destaque no centro da figura, uma vez que ele é a eixo propulsor de um curso e consequentemente do PDD. No exercício de qualquer uma das suas atividades ele é constantemente avaliador. Os professores pesquisados reconhecem a avaliação formativa como um importante instrumento de gestão, porém destacam que ela não tem sido utilizada de forma efetiva. Acredita-se que se a gestão estivesse utilizando a avaliação como instrumento formativo dos atores envolvidos e do processo de ensino/aprendizagem, talvez nesta pesquisa, não teriam aparecido tantas fragilidades relativas à gestão, o que possivelmente permitiria maior sustentabilidade do PDD. 
Figura 1 - Representação das atividades do gestor do curso médico nos Métodos Ativos.

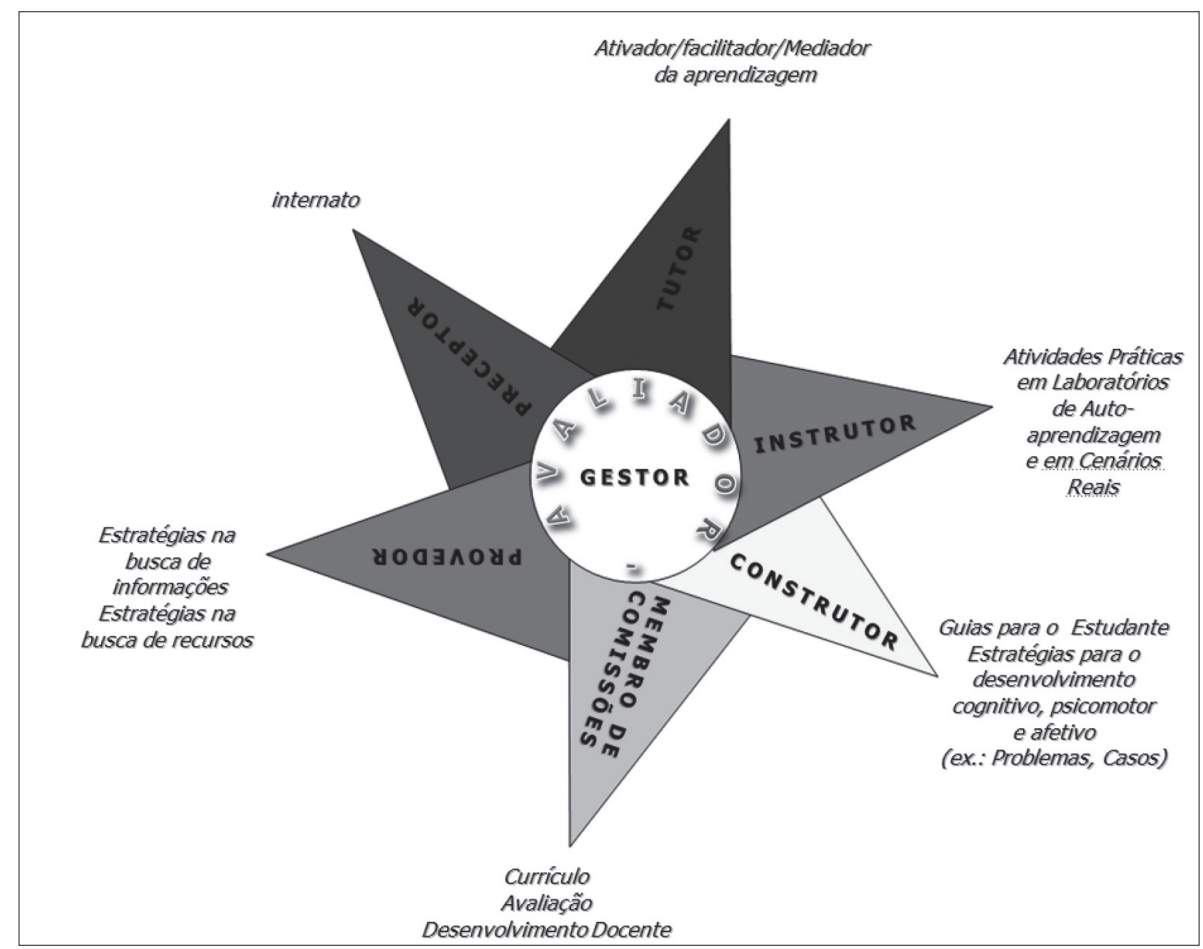

Fonte: Adaptada de: ALMEIDA, Maria Tereza Carvalho; BATISTA, Nildo Alves. Ser docente em métodos ativos de ensino/aprendizagem na formação do médico. Revista Brasileira de Educação Médica, Rio de Janeiro, v. 35, n. 4, 2011.

\section{REFERÊNCIAS}

ALMEIDA, Henriqueta Galvanin Guidio et al. Elaborando a capacitação de tutores: subsídios da prática. Revista Brasileira de Educação Médica, Rio de Janeiro, v. 27, p. 191-198, 2003.

ALMEIDA, Márcio José de. Gestão na escola médica: crítica e autocrítica. Revista Brasileira de Educação Médica, Rio de Janeiro, v. 32, p. 202-209, 2008. 
ALMEIDA, Maria Tereza Carvalho; BATISTA, Nildo Alves. Ser docente em métodos ativos de ensino/aprendizagem na formação do médico. Revista Brasileira de Educação Médica, Rio de Janeiro, v. 35, n. 4, 2011.

BATISTA, Nildo Alves; BATISTA, Sylvia Helena Souza da Silva. Docência em saúde: temas e experiências. São Paulo: Senac, 2004.

BOGDAN, Roberto; BIKLEN, Sari. Investigação qualitativa em educação: uma introdução à teoria e aos métodos, 1994. (Coleção ciências da educação).

CAMPOS, Henry de Holanda et al. Programas de desenvolvimento docente em escolas médicas: oportunidades e perspectivas. Mais do que uma necessidade. Revista Brasileira de Educação Médica, Rio de Janeiro, v. 3, p. 34-38, 2007.

COSTA, Nilce Maria da Silva Campos. Formação pedagógica de professores de medicina. Revista Latino-Americana de Enfermagem, Ribeirão Preto, v. 18, n. 1, [07 telas], jan/fev/2010.

CUNHA, Maria Izabel. O professor universitário na transição de paradigmas. Araraquara: JM, 1998.

HOFFMANN, Jussara. Avaliar para promover: as setas do caminho. 27 ed. Porto Alegre: Meditação, 2008.

IOCHIDA, Lúcia Christina. Metodologias problematizadoras no ensino em saúde. In: BATISTA, Nildo Alves; BATISTA, Sylvia Helena Souza da Silva. Docência em saúde: temas e experiências. São Paulo: Senac, 2004.

MINAYO, Maria Cecília de Souza. O desafio do conhecimento-pesquisa qualitativa em saúde. São Paulo: Hucitec, 2004.

PASQUALI, Luiz (Org.) Teoria e métodos de medida em ciências do comportamento. Brasília (DF): Laboratório de Pesquisa em Avaliação e Medida. Instituto de Psicologia. UNB, INEP, 1996.

PERIM, Gianna Lepre et al. Desenvolvimento docente e a formação dos médicos. Revista Brasileira de Educação Médica, Rio de Janeiro, v. 33, p. 70-82, 2009.

RUSHTON, Alison. Formative assessment: a key to deep learning? Medical Teacher, Reino Unido, v. 27, n. 6, p. 509-513. 2005. 
SILVA, Janssen Felipe. Avaliação na perspectiva formativa-reguladora. Pressupostos teóricos e práticos. 2. ed. Porto Alegre: Mediação, 2006. TSUJI, Hissachi; AGUILAR-DA-SILVA, Rinaldo Henrique. Aprender e ensinar na escola vestida de branco: do modelo biomédico ao humanístico. São Paulo: Forte, 2010.

VENTURELLI José; FIORINI Vânia Maria. Programas Educacionais Inovadores em Escolas Médicas: Capacitação Docente. Revista Brasileira de Educação Médica, Rio de Janeiro, v. 25, p. 7-21, 2001.

WILKERSON, LuAnn; IRBY, David M. Strategies for improving teaching pratices: a compreensive aproach to faculty developement. Acad. Med, v. 73; p. 387-396, 1998. 